\title{
Hawking, fiducial, and free-fall temperature of black hole on gravity's rainbow
}

\author{
Yongwan Gim ${ }^{\mathrm{a}}$, Wontae Kim ${ }^{\mathrm{b}}$ \\ Department of Physics, Sogang University, Seoul 121-742, Republic of Korea
}

Received: 24 December 2015 / Accepted: 15 March 2016 / Published online: 25 March 2016

(C) The Author(s) 2016. This article is published with open access at Springerlink.com

\begin{abstract}
On gravity's rainbow, the energy of test particles deforms the geometry of a black hole in such a way that the corresponding Hawking temperature is expected to be modified. It means that the fiducial and free-fall temperatures on the black hole background should also be modified according to deformation of the geometry. In this work, the probing energy of test particles is assumed as the average energy of the Hawking particle in order to study the particle back reaction of the geometry by using the advantage of gravity's rainbow. We shall obtain the modified fiducial and free-fall temperatures, respectively. The behaviors of these two temperatures on the horizon tell us that black hole complementarity is still well defined on gravity's rainbow.
\end{abstract}

\section{Introduction}

There has been given much attention to modified dispersion relations in the regime of gravity's rainbow from the semiclassical point of view of loop quantum gravity [1-5]. Such modifications could be found in threshold anomalies in ultrahigh cosmic rays and Tev photons [6-13]; however, they are still not established. Moreover, threshold anomalies are not a generic feature of the modified dispersion relation but they are only predicted by modified dispersion relation scenarios with a preferred reference frame [14]. In fact, the modified dispersion relations were based on the doubly special relativity [15-20], which is an extended version of Einstein's special relativity in the sense that both the Planck length and the speed of light should be required to be invariant in any inertial frames. In connection with this issue, it was claimed that a nonlinear Lorentz transformation in the momentum space is needed to keep the double invariant constants. Subsequently, Magueijo and Smolin [21] proposed that the spacetime background felt by a test particle depends on its energy

\footnotetext{
a e-mail: yongwan89@ sogang.ac.kr

be-mail: wtkim@sogang.ac.kr
}

such that the energy of the test particle deforms the background geometry and eventually gives modified dispersion relations. In particular, according to the modified dispersion relations with the generalized uncertainty principle, it was shown that the generalized second law of black hole thermodynamics is valid by modifying a relation between the mass and temperature of the black hole [22]. Moreover, it was proposed that the brick wall could be eliminated by choosing appropriate rainbow functions [23], and also claimed that a remnant is formed for all black objects in this theory [24]. The gravity's rainbow has been extensively studied in order to explore various aspects for black holes and cosmology [25-38].

One of the most important ingredients in thermodynamic analysis of black hole system is to define temperatures consistently [39]. The Hawking temperature could be defined by the surface gravity $\kappa(E)$ from the metric of black holes on gravity's rainbow [40], and thus the metric would naturally depend on the energy $E$ of the test particle in terms of the rainbow functions to modify the dispersion relation. In the spirit of gravity's rainbow, any probing energy affects the geometry, so it seems plausible to assert that Hawking radiation deforms the original background geometry. Hence, if the temperature were regarded as the average energy of test particles on the background of black hole, then the Hawking temperature should be characterized by the deformed geometry. Its form would be different from the standard Hawking temperature due to the rainbow effect. Apart from the Hawking temperature defined at infinity, one can also consider additional two different temperatures; the so-called fiducial temperature and free-fall temperature. The former is defined in fixed coordinates of an accelerated frame, while the latter is defined in a free-falling frame. We expect these two temperatures would be modified like the Hawking temperature according to the rainbow effect, and thus it would be interesting to study how to obtain the fiducial temperature and 
free-fall temperature on the background of a black hole in the theory of gravity's rainbow.

In Sect. 2, we shall elaborate the Hawking temperature on gravity's rainbow. At first sight, the temperature obtained from the surface gravity in the energy-independent coordinates seems different from that from the energy-dependent coordinates. To resolve this conflict, we will find a useful relation between these two temperatures. Additionally, we shall identify the relation between the energy of test particles and the Hawking temperature. Then a proportional constant between the energy and the temperature will be fixed by using the modified dispersion relation and the uncertainty relation. In Sect. 3, our strategy for the calculation of the fiducial temperature is to use the conventional definition, but written in terms of the energy-dependent coordinates [21]. For a certain class of rainbow functions, we obtain an explicit fiducial temperature which becomes the Hawking temperature at infinity. On the other hand, the free-fall temperature may be derived by employing the Stefan-Boltzmann relation to relate the energy density with the temperature in a free-falling frame. Unfortunately, this is not the case, since the conventional Stefan-Boltzmann relation in the proper frame [41,42] is not appropriate to apply it directly to quantum black holes, because it was obtained by assuming traceless condition of the energy-momentum tensor. So the free-fall temperature gives a pathological behavior at the horizon as discussed in detail in Ref. [43]. In Sect. 4, we present the generalized Stefan-Boltzmann relation for a non-vanishing trace in the presence of Hawking radiation. In fact, Hawking radiation is related to the trace anomaly of the energy-momentum tensors [44]. Then the free-fall energy density and the free-all temperature will be calculated on gravity's rainbow. In Sect. 5 , conclusion and discussion will be given.

\section{Hawking temperature}

Let us start with the modified dispersion relation [21]

$E^{2} f\left(E / E_{p}\right)^{2}-p^{2} g\left(E / E_{p}\right)^{2}=m^{2}$,

where $E, p, m$ are the energy, momentum, mass of a test particle, and the Planck energy is denoted by $E_{p}$. We use the natural units as $\hbar=c=k_{\mathrm{B}}=1$. The rainbow functions $f\left(E / E_{p}\right), g\left(E / E_{p}\right)$ satisfy the limits of $\lim _{E / E_{p} \rightarrow 0} f\left(E / E_{p}\right)=1$ and $\lim _{E / E_{p} \rightarrow 0} g\left(E / E_{p}\right)=1$. Note that the above modified dispersion relation can be rewritten in the form of the original dispersion relation such as $\tilde{E}^{2}-\tilde{p}^{2}=m^{2}$ by using the transformation,

$\tilde{E}=f(E) E, \quad \tilde{p}=g(E) p$.

From now on, we are going to use a two-dimensional metric in order for exact solubility without losing the essential properties of temperatures. So let us consider the Schwarzschild black hole on gravity's rainbow,

$\mathrm{d} s^{2}=-F_{1}(r, E) \mathrm{d} t^{2}+F_{2}(r, E) \mathrm{d} r^{2}$,

where the metric functions are $F_{1}(r, E)=f^{-2}(E)(1-$ $2 G M / r)$ and $F_{2}(r, E)=g^{-2}(E)(1-2 G M / r)^{-1}$. Then the Hawking temperature can be obtained from the surface gravity as [40]

$$
\begin{aligned}
T_{\mathrm{H}} & =\frac{\kappa_{\mathrm{H}}}{2 \pi} \\
& =\left.\frac{1}{2 \pi} \sqrt{-\frac{1}{2} \nabla^{\mu} \xi^{\nu} \nabla_{\mu} \xi_{\nu}}\right|_{r=r_{\mathrm{H}}} \\
& =\frac{g\left(E / E_{p}\right)}{f\left(E / E_{p}\right)} \frac{1}{8 \pi G M},
\end{aligned}
$$

where $\xi_{\mu}$ is the time-like Killing vector and $r_{H}$ is the event horizon.

On the other hand, the metric (3) can also be written in terms of the energy-dependent coordinates as [21]

$\mathrm{d} s^{2}=-\left(1-\frac{2 \tilde{G} M}{\tilde{r}}\right) \mathrm{d} \tilde{t}^{2}+\frac{1}{1-\frac{2 \tilde{G} M}{\tilde{r}}} \mathrm{~d} \tilde{r}^{2}$,

which the transformation is implemented by $\tilde{t}(E)=t / f(E)$, $\tilde{r}(E)=r / g(E)$, and $\tilde{G}(E)=G / g(E)$, where the tilde variables are energy-dependent. From the metric (7), the Hawking temperature can be derived from the definition of the surface gravity as

$\tilde{T}_{\mathrm{H}}=\frac{1}{8 \pi \tilde{G} M}$.

Note that it can be shown that the temperature (8) is the same as Eq. (6) if the temperature transformation is assumed as

$\tilde{T}_{\mathrm{H}}=f(E) T_{\mathrm{H}}$,

which yields a result compatible with Eq. (6) after rewriting it in terms of the non-tilde variables,

$T_{\mathrm{H}}=\frac{g\left(E / E_{p}\right)}{f\left(E / E_{p}\right)} \frac{1}{8 \pi G M}$.

From the transformation (9) and the first equation in Eq. (2), one can see that the temperature $T_{\mathrm{H}}$ behaves in the same manner as the energy $E$ on gravity's rainbow. According to this fact, it is reasonable to relate the temperature to the energy. If the Hawking radiation were regarded as the energy of test particles, then the framework of gravity's rainbow would provide the particle back reaction of the geometry effectively and, consequently, modify the standard Hawking temperature. However, the test particles with different energies would give different geometries. So let us choose a single representative energy, that is, the average energy of particles. 
Thus it will be proportional to the Hawking temperature of thermal bath based on Wien's law [40],

$E=\alpha T_{\mathrm{H}}$,

where $\alpha$ is a proportional constant. On general grounds, it seems non-trivial to determine the constant. Nevertheless, we shall fix the constant for a specific modified dispersion relation such as $[45,46]$

$m^{2}=E^{2}-p^{2}+\eta p^{2}\left(\frac{E}{E_{p}}\right)^{n}$,

where $\eta$ is a positive rainbow parameter and $n$ is a positive integer. Then the rainbow functions can be read off from Eq. (12) [34],

$f\left(E / E_{p}\right)=1, \quad g\left(E / E_{p}\right)=\sqrt{1-\eta\left(\frac{E}{E_{p}}\right)^{n}}$,

where $n=2$ for simplicity.

Following the argument in Ref. [22], the Heisenberg uncertainty relation can be used to obtain the momentum of the particle as $p=\Delta p \sim 1 /(2 G M)$, where the position uncertainty of the particle is $\Delta x \sim 2 G M$. So the energy can be expressed as $E=\sqrt{\left(1+4 m^{2} G^{2} M^{2}\right)\left(\eta G+4 G^{2} M^{2}\right)^{-1}}$ with $G=1 / E_{p}^{2}$. From Eq. (11), the temperature can be identified as

$T_{\mathrm{H}}=\frac{1}{2 \alpha G M} \sqrt{\frac{4 G M^{2}+16 m^{2} G^{3} M^{4}}{4 G M^{2}+\eta}}$.

Next, using the rainbow functions (13) and the energytemperature relation (11), the temperature defined by the surface gravity (10) can be fixed as

$T_{\mathrm{H}}=\frac{1}{8 \pi G M} \sqrt{\frac{64 \pi^{2} G M^{2}}{64 \pi^{2} G M^{2}+\alpha^{2} \eta}}$.

Note that the Hawking temperature (15) defined at infinity by using the surface gravity method was originally obtained by assuming a massless scalar field [39]. For the massive case (14), we are actually interested in the case for the welldefined semiclassical approximations for which $m M \ll 1$. In fact, the massive modes will propagate near infinity but it will decay exponentially there. Furthermore, the constant $\alpha$ should depend on the mass of particle $m$ and the mass of black hole $M$, such that it cannot be an universal constant any more. In these respects, it is reasonable to take the massless limit for simplicity in order to compare Eqs. (14)-(15). Then the proportional constant $\alpha$ is uniquely fixed as $\alpha=4 \pi$.

After all, the temperature (10) can be expressed as

$T_{\mathrm{H}}=\frac{1}{8 \pi G M} \sqrt{\frac{4 G M^{2}}{4 G M^{2}+\eta}}$,

which respects the well-known Hawking temperature for $\eta \rightarrow 0$. Note that the above result is different from Eq. (10) in general; however, they are the same if the energy of probing particles is the average energy of Hawking particles.

In contrast to the standard Hawking temperature, the Hawking temperature (16) on gravity's rainbow is finite when the mass of black hole vanishes thanks to the rainbow parameter $\eta$, which plays the role of a cutoff. In the subsequent sections, we will investigate the fiducial temperature and the free-fall temperature by using the energy-temperature relation (11) and Hawking temperature on gravity's rainbow (16).

\section{Fiducial temperature}

The fiducial temperature for the fixed observer in an accelerated frame on a black hole can be expressed in the form of blue-shifted Hawking temperature by using the time dilation of frequency at different places [47]. So, from the metric (7), the fiducial temperature can be written as

$\tilde{T}_{\mathrm{FID}}=\frac{\tilde{T}_{\mathrm{H}}}{\sqrt{-\tilde{g}_{t t}}}$.

Note that at $\tilde{r} \rightarrow \infty, \tilde{T}_{\mathrm{FID}}=\tilde{T}_{\mathrm{H}}$, which means that the fiducial temperature is also the same transformation rule as

$\tilde{T}_{\mathrm{FID}}=f(E) T_{\mathrm{FID}}$

like Eq. (9). Along with $\tilde{G}=G / g(E)$ and $\tilde{r}=r / g(E)$, one can easily obtain the fiducial temperature as

$T_{\mathrm{FID}}=\frac{g(E)}{f(E)} \frac{1}{8 \pi G M \sqrt{1-\frac{2 G M}{r}}}$,

and the specific choice of rainbow functions (13) gives

$T_{\mathrm{FID}}=\sqrt{1-\eta\left(\frac{E}{E_{p}}\right)^{2}} \frac{1}{8 \pi G M \sqrt{1-\frac{2 G M}{r}}}$.

In the previous section, we identified the energy of particle as the Hawking temperature. So, plugging $E=4 \pi T_{H}$ into the fiducial temperature (20), one can explicitly write it as

$$
\begin{aligned}
T_{\mathrm{FID}} & =\sqrt{1-\eta\left(\frac{4 \pi T_{\mathrm{H}}}{E_{p}}\right)^{2}} \frac{1}{8 \pi G M \sqrt{1-\frac{2 G M}{r}}} \\
& =\frac{1}{8 \pi G M \sqrt{1-\frac{2 G M}{r}}} \sqrt{\frac{4 G M^{2}}{4 G M^{2}+\eta}}
\end{aligned}
$$

where we also used Eq. (16). Thus we can show that the fiducial temperature on gravity's rainbow is simply given as the blue-shifted Hawking temperature i.e., $T_{\mathrm{FID}}=$ 


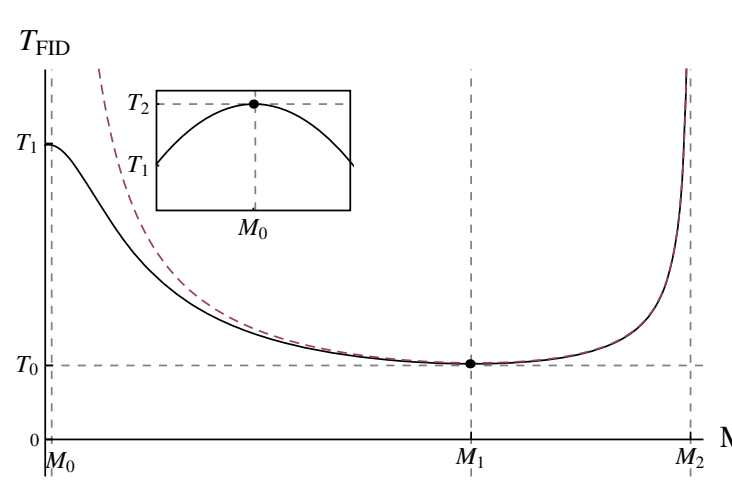

(a) $T_{\text {FID }}(M)$ with fixed $r$

Fig. 1 We set $G=1, r=20, M_{2}=10$ in (a), and $M=1$ in (b). The solid curves are for the fiducial temperatures on gravity's rainbow $(\eta=1)$ and the dashed curves are for the conventional fiducial temperatures $(\eta=0)$. From the small box in a, one can see that the fiducial

$T_{\mathrm{H}} / \sqrt{1-2 G M / r}$. In Fig. 1a, b, the overall behaviors of the fiducial temperature on gravity's rainbow are plotted in contrast to the conventional ones.

\section{Free-fall temperature}

In this section, we will derive the free-fall temperature defined by a free-falling observer dropped from rest. First of all, it is worth to note that the energy density in the free-falling frame should be rephrased by the temperature through the Stefan-Boltzmann relation which relates the energy density in the proper frame to the free-fall temperature. In fact, by using the Stefan-Boltzmann relation, the free-fall temperature called the Tolman temperature [41,42] was obtained assuming the traceless condition of the energymomentum tensor. Thus the conventional Stefan-Boltzmann relation should be generalized in such a way to incorporate the trace anomaly of energy-momentum tensor [48], since Hawking radiation is associated with the trace anomaly [44].

For this purpose, we repeat the calculation along the line of the original work by Tolman $[41,42]$ except the traceless condition of energy-momentum tensor. From the first law of thermodynamics given as $\mathrm{d} U=T \mathrm{~d} S-p \mathrm{~d} V$, where $U, T$, $S, p$, and $V$ are the thermodynamic internal energy, temperature, entropy, pressure, and volume in the proper frame, respectively, and $U=\int \rho \mathrm{d} V$, one can get

$$
\left.\frac{\partial U}{\partial V}\right|_{T}=\left.T \frac{\partial S}{\partial V}\right|_{T}-p .
$$

By employing the Maxwell relation such as $\partial S /\left.\partial V\right|_{T}=$ $\partial p /\left.\partial T\right|_{V}$, Eq. (23) is rewritten as

$\rho=\left.T \frac{\partial p}{\partial T}\right|_{V}-p$

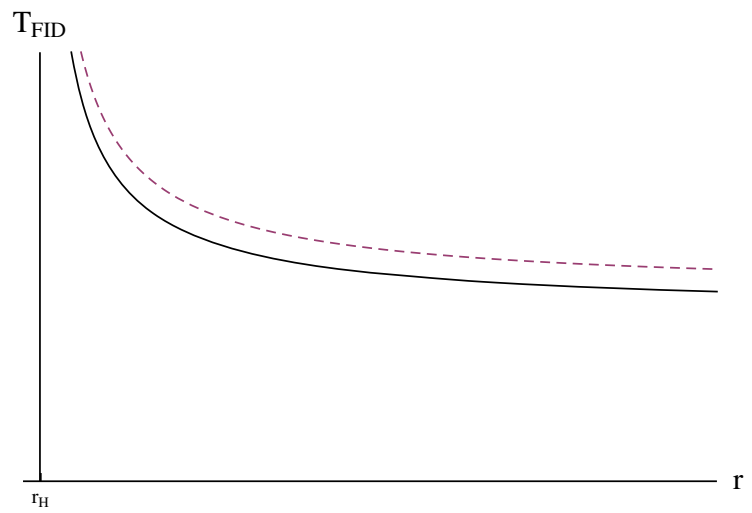

(b) $T_{\text {FID }}(r)$ with fixed $M$

temperature has a finite maximum $T_{2}$ at $M_{0}$ and is terminated when $M \rightarrow 0$. In $\mathbf{b}$, the value of the fiducial temperature is smaller than the conventional one, and it is still divergent at the horizon

Next the trace of energy-momentum tensor is expressed as

$-\rho+p=T_{\mu}^{\mu}$.

Plugging Eqs. (25) into (24) along with the property of the temperature independence of the trace anomaly [49], one can get

$2 \rho=\left.T \frac{\partial \rho}{\partial T}\right|_{V}-T_{\mu}^{\mu}$,

which yields the following solutions:

$\rho=\gamma T^{2}-\frac{1}{2} T_{\mu}^{\mu}, \quad p=\gamma T^{2}+\frac{1}{2} T_{\mu}^{\mu}$,

where the Stefan-Boltzmann constant is chosen as $\gamma=\pi / 6$ for a two-dimensional massless scalar field [44]. This is the generalized Stefan-Boltzmann relation to incorporate the effect of the trace anomaly. As it should be, it reproduces the conventional Stefan-Boltzmann relation when the energymomentum tensor is traceless.

On the other hand, we are now in a position to derive the free-fall energy density and the pressure. In a static system, the Hawking radiation can be treated as a perfect fluid [41],

$T^{\mu \nu}=(\rho+p) u^{\mu} u^{v}+p g^{\mu \nu}$,

and from the metric (3) the velocity for the free-falling observer is solved as

$u^{\mu}=\frac{\mathrm{d} x^{\mu}}{\mathrm{d} \tau}=\left(\frac{1}{\sqrt{F_{1}(r, E)}}, 0\right)$.

Note that we assumed the Hawking radiation as a perfect fluid on the static background of black hole. Of course, the background satisfies the equation of motion from gravity's rainbow without source, whereas the excitations such as Hawking particles are treated as quantized particles. Actually, we are interested in the semiclassical limit, such that the background 


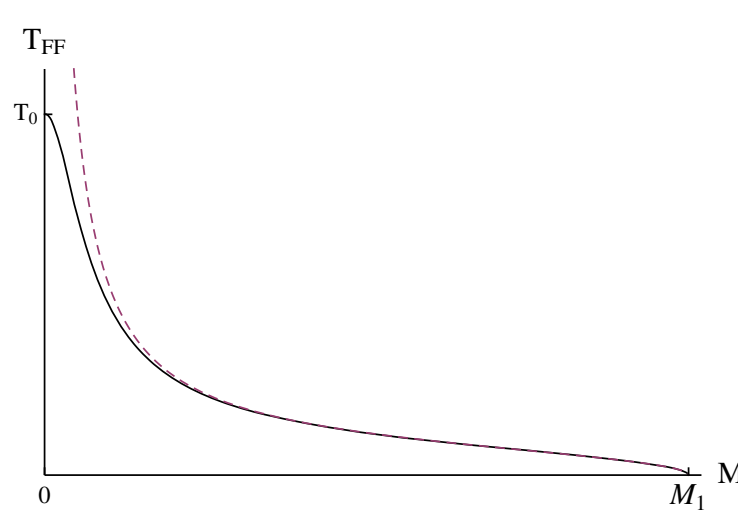

(a) $T_{\mathrm{FF}}(M)$ with fixed $r$

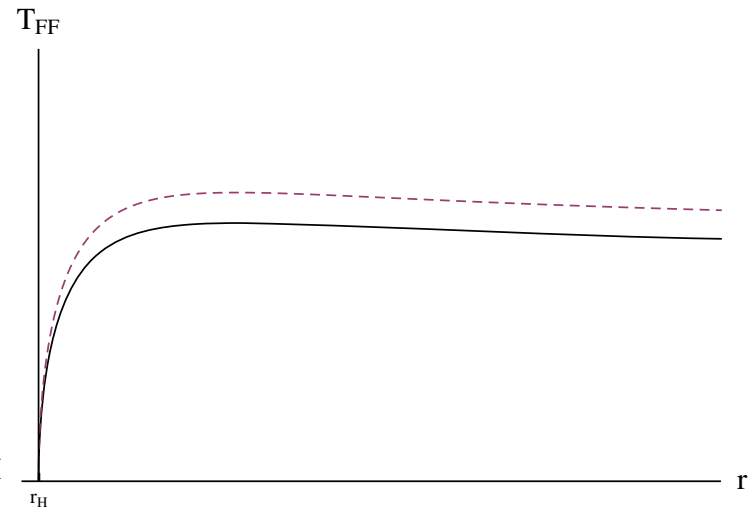

(b) $T_{\mathrm{FF}}(r)$ with fixed $M$

Fig. 2 We set $G=1, r=20, M_{1}=10$ in (a), and $M=1$ in (b). The solid curves are for the temperature on gravity's rainbow, which are plotted by setting $\eta=1$ for convenience, and the dashed curves are for the conventional temperature without the rainbow effect, simply setting $\eta=0$

is indeed classically vacuum solution whereas the test particles or radiation are quantized by means of the quantum energy-momentum tensor on this classical background.

Now, the free-fall energy density and the pressure can be related to the quantities defined in the fixed coordinates in terms of the relations; $\rho=T_{\mu \nu} u^{\mu} u^{\nu}, p=T_{\mu \nu} n^{\mu} n^{\nu}$, where $n^{\mu}$ is the spacelike unit normal vector satisfying $n^{\mu} n_{\mu}=1$ and $n^{\mu} u_{\mu}=0$. Then the covariant conservation law of the energy-momentum tensor can be written in the form of

$2 F_{1} \partial_{r} p=-(\rho+p) \partial_{r} F_{1}$

By using the trace equation (25), the solution to the differential equation (30) is solved as

$\rho=\frac{1}{F_{1}}\left(C_{0}-F_{1} T_{\mu}^{\mu}+\frac{1}{2} \int T_{\mu}^{\mu} \mathrm{d} F_{1}\right)$,

$p=\frac{1}{F_{1}}\left(C_{0}+\frac{1}{2} \int T_{\mu}^{\mu} \mathrm{d} F_{1}\right)$,

where $C_{0}$ is an integration constant. Plugging the energy density and pressure (31) into Eq. (27), we can obtain the generalized Tolman temperature as

$T_{\mathrm{FF}}=\frac{1}{\sqrt{\gamma F_{1}}} \sqrt{C_{0}-\frac{F_{1}}{2} T_{\mu}^{\mu}+\frac{1}{2} \int T_{\mu}^{\mu} \mathrm{d} F_{1}}$.

For the traceless case, the temperature is reduced to the standard Tolman temperature, $T_{\mathrm{FF}}=\sqrt{C_{0} /\left(\gamma F_{1}\right)}$.

Let us calculate the free-fall temperature for the twodimensional Schwarzschild black hole described by the metric (3). By using the trace anomaly for a massless scalar field as $T_{\mu}^{\mu}=R /(24 \pi)$ [44,50], the trace anomaly for the metric (3) is obtained:

$T_{\mu}^{\mu}=g(E)^{2} \frac{G M}{6 \pi r^{3}}$.

From Eqs. (32) and (33), the free-fall temperature can be obtained with the boundary condition of $C_{0}=\gamma T_{\mathrm{H}}^{2}$, in which the standard Hawking temperature is restored at infinity,

$$
\begin{aligned}
T_{\mathrm{FF}}= & \frac{1}{8 \pi G M} \sqrt{\frac{4 G M^{2}}{4 G M^{2}+\eta}} \\
& \times \sqrt{1+\frac{2 G M}{r}+\left(\frac{2 G M}{r}\right)^{2}-3\left(\frac{2 G M}{r}\right)^{3}} .
\end{aligned}
$$

The behaviors of the free-fall temperature are plotted in Fig. 2a, b. First of all, without the rainbow effect taking $\eta \rightarrow 0$, the temperature is divergent for the massless limit of the black hole due to the rapid evaporation of black hole but the rainbow parameter cuts off the divergence as seen from Fig. 2(a). On the other hand, from Fig. 2(b), one can see that the temperature becomes the Hawking temperature on gravity's rainbow at infinity, while it vanishes at the horizon. The radial dependence of the free-fall temperature shows that the rainbow effect lowers the value of the free-fall temperature.

\section{Conclusion and discussion}

The energy of probing particles affects the geometry in the formalism of gravity's rainbow, which is comparable to take into account the test particle back reaction of the geometry. It means that the energy of particle modifies the geometry of black hole, so that the Hawking temperature, which is sensitive to the geometry of black hole, can also be modified. In this context, we studied the Hawking, fiducial, and free-fall temperatures, respectively, in order to obtain their characteristics in the framework on gravity's rainbow.

For the Hawking temperature, we presented two representations in the fashion of the energy-independent and dependent coordinates, and found that the relation to connect these representations follows $\tilde{T}=f T$ like the energy transformation. Moreover, the Hawking radiation was iden- 
tified with using the energy of test particles in order to investigate the impact on the geometry in the presence of radiation. We found that the energy-temperature relation was specified as $E=\alpha T_{\mathrm{H}}$, where $\alpha=4 \pi$ in our choice of rainbow functions. It is interesting to note that the proportional constant was given by the irrational number, which is contrast to the common case given as multiple degrees of half-integer. It means that the particle energy should be nontrivially related to the thermal temperature. Next, the fiducial temperature was defined by using the energy-dependent coordinates, and then it was rewritten in terms of the energyindependent coordinates. Using the energy-temperature relation, we found that the fiducial temperature (21) takes the blue-shifted Hawking temperature that is still divergent at the horizon, while it reproduces the Hawking temperature at infinity. For the free-fall temperature, we extended the conventional Stefan-Boltzmann relation to the case of the nonvanishing trace of energy-momentum tensor in order to take into account the trace anomaly related to Hawking radiation. Consequently, the free-fall temperature is finite everywhere without the blueshift, especially vanishing at the horizon.

In connection with the last statement, one might want to find a different reason why the free-fall temperature (34) vanishes at the horizon, whereas the fiducial temperature (21) is divergent there. This fact can also be seen from the Unruh effect [51] for the large black hole. Very near the horizon, the metric (7) can be written as the Rindler metric, so that the acceleration of the fixed observer is proportional to the temperature as $\tilde{T}_{\mathrm{U}}=\tilde{a} / 2 \pi$. By recovering it in the energy-independent coordinates, the Unruh temperature on gravity's rainbow can be written as $T_{\mathrm{U}}=$ $(g / f) G M /\left(2 \pi r^{2} \sqrt{1-2 G M / r}\right)$. From the choice of rainbow functions such as Eq. (13) with Eq. (11), we can see that it should be divergent at the horizon, which is coincident with the present result for the fiducial observer. As a corollary in the local inertial frame, there does not exist any acceleration at the horizon for the large black hole, so that the Unruh temperature vanishes. It means that our free-fall temperature should be zero at the horizon. Thus the fixed observer and free-fall observer see extremely different degrees of freedom at the horizon, which means that black hole complementarity [52-54] still holds for gravity's rainbow.

The final comment is in order. At first sight, the freefall temperature generically seems to vanish in freely falling frames at any distance far from the horizon including at the horizon because of the equivalence principle. However, this is not the case, since there exists an energy density which amounts to the curvature scale of $1 / M^{2}$ even in those frames in which the gravitational acceleration is locally zero. Thus, the scale of the corresponding temperature is the order of $1 / M$ as seen from Eq. (27) rather than zero. Therefore, the common wisdom is that the equivalence principle is weakly broken when the Hawking radiation is involved quantum- mechanically. However, the surprise is that, as shown in Ref. [55], the equivalence principle is restored just at the horizon. For large black holes, this fact is compatible with the Unruh effect which was actually defined at the horizon for those black holes in the Rindler approximation. In the present calculations, this vanishing result of the free-fall temperature at the horizon was found from the modification of the Stefan-Boltzmann law by taking into account the trace anomaly.

Acknowledgments We would like to thank M. Eune for helpful comments. This work was supported by the National Research Foundation of Korea (NRF) Grant funded by the Korea government (MSIP) (2014R1A2A1A11049571).

Open Access This article is distributed under the terms of the Creative Commons Attribution 4.0 International License (http://creativecomm ons.org/licenses/by/4.0/), which permits unrestricted use, distribution, and reproduction in any medium, provided you give appropriate credit to the original author(s) and the source, provide a link to the Creative Commons license, and indicate if changes were made.

Funded by SCOAP S $^{3}$

\section{References}

1. R. Gambini, J. Pullin, Nonstandard optics from quantum spacetime. Phys. Rev. D 59, 124021 (1999). arXiv:gr-qc/9809038

2. J. Alfaro, H.A. Morales-Tecotl, L.F. Urrutia, Loop quantum gravity and light propagation. Phys. Rev. D 65, 103509 (2002). arXiv:hep-th/0108061

3. H. Sahlmann, T. Thiemann, Towards the QFT on curved space-time limit of QGR. 2. A concrete implementation. Class. Quant. Grav. 23, 909-954 (2006). arXiv:gr-qc/0207031

4. L. Smolin, Quantum gravity with a positive cosmological constant. (2002) arXiv:hep-th/0209079

5. L. Smolin, Falsifiable predictions from semiclassical quantum gravity. Nucl. Phys. B 742, 142-157 (2006). arXiv:hep-th/0501091

6. G. Amelino-Camelia, J.R. Ellis, N.E. Mavromatos, D.V. Nanopoulos, S. Sarkar, Tests of quantum gravity from observations of gamma-ray bursts. Nature 393, 763-765 (1998). arXiv:astro-ph/9712103

7. G. Amelino-Camelia, J. Lukierski, A. Nowicki, kappa deformed covariant phase space and quantum gravity uncertainty relations. Phys. Atom. Nucl. 61, 1811-1815 (1998). arXiv:hep-th/9706031

8. D. Colladay, V.A. Kostelecky, Lorentz violating extension of the standard model. Phys. Rev. D 58, 116002 (1998). arXiv:hep-ph/9809521

9. S.R. Coleman, S.L. Glashow, High-energy tests of Lorentz invariance. Phys. Rev. D 59, 116008 (1999). arXiv:hep-ph/9812418

10. G. Amelino-Camelia, J. Lukierski, A. Nowicki, Distance measurement and kappa deformed propagation of light and heavy probes. Int. J. Mod. Phys A 14, 4575-4588 (1999). arXiv:gr-qc/9903066

11. G. Amelino-Camelia, T. Piran, Planck scale deformation of Lorentz symmetry as a solution to the UHECR and the TeV gamma paradoxes. Phys. Rev. D 64, 036005 (2001). arXiv:astro-ph/0008107

12. T. Jacobson, S. Liberati, D. Mattingly, $\mathrm{TeV}$ astrophysics constraints on Planck scale Lorentz violation. Phys. Rev. D 66, 081302 (2002). arXiv:hep-ph/0112207

13. T.A. Jacobson, S. Liberati, D. Mattingly, F.W. Stecker, New limits on Planck scale Lorentz violation in QED. Phys. Rev. Lett. 93, 021101 (2004). arXiv:astro-ph/0309681 
14. G. Amelino-Camelia, Phenomenology of Planck-scale Lorentz-symmetry test theories. New J. Phys. 6, 188 (2004). arXiv:gr-qc/0212002

15. G. Amelino-Camelia, Testable scenario for relativity with minimum length. Phys. Lett. B 510, 255-263 (2001). arXiv:hep-th/0012238

16. G. Amelino-Camelia, Relativity in space-times with short distance structure governed by an observer independent (Planckian) length scale. Int. J. Mod. Phys D 11, 35-60 (2002). arXiv:gr-qc/0012051

17. G. Amelino-Camelia, The Three perspectives on the quantum gravity problem and their implications for the fate of Lorentz symmetry, in On recent developments in theoretical and experimental general relativity, gravitation, and relativistic field theories. Proceedings, 10th Marcel Grossmann Meeting, MG10, Rio de Janeiro, July 20 26, 2003. Pt. A-C, pp. 2214-2216. (2003). arXiv:gr-qc/0309054

18. G. Amelino-Camelia, J. Kowalski-Glikman, G. Mandanici, A. Procaccini, Phenomenology of doubly special relativity. Int. J. Mod. Phys. A 20, 6007-6038 (2005). arXiv:gr-qc/0312124

19. J. Magueijo, L. Smolin, Lorentz invariance with an invariant energy scale. Phys. Rev. Lett. 88, 190403 (2002). arXiv:hep-th/0112090

20. J. Magueijo, L. Smolin, Generalized Lorentz invariance with an invariant energy scale. Phys. Rev. D 67, 044017 (2003). arXiv:gr-qc/0207085

21. J. Magueijo, L. Smolin, Gravity's rainbow, Class. Quant. Grav. 21, 1725-1736 (2004). arXiv:gr-qc/0305055

22. G. Amelino-Camelia, M. Arzano, Y. Ling, G. Mandanici, Blackhole thermodynamics with modified dispersion relations and generalized uncertainty principles, Class. Quant. Grav. 23, 2585-2606 (2006). arXiv:gr-qc/0506110

23. R. Garattini, Modified dispersion relations and black hole entropy. Phys. Lett. B 685, 329-337 (2010). arXiv:0902.3927

24. A.F. Ali, M. Faizal, M.M. Khalil, Remnant for all black objects due to gravity's rainbow. Nucl. Phys. B 894, 341-360 (2015). arXiv: 1410.5706

25. P. Galan, G.A. Mena Marugan, Quantum time uncertainty in a gravity's rainbow formalism. Phys. Rev. D 70, 124003 (2004). arXiv:gr-qc/0411089

26. J. Hackett, Asymptotic flatness in rainbow gravity. Class. Quant. Grav 23, 3833-3842 (2006). arXiv:gr-qc/0509103

27. R. Aloisio, A. Galante, A. Grillo, S. Liberati, E. Luzio, F. Mendez, Deformed special relativity as an effective theory of measurements on quantum gravitational backgrounds. Phys. Rev. D 73, 045020 (2006). arXiv:gr-qc/0511031

28. P. Galan, G.A. Mena, Marugan, Entropy and temperature of black holes in a gravity's rainbow. Phys. Rev. D 74, 044035 (2006). arXiv:gr-qc/0608061

29. G. Amelino-Camelia, M. Arzano, G. Gubitosi, J. Magueijo, Rainbow gravity and scale-invariant fluctuations. Phys. Rev. D 88, 041303 (2013). arXiv:1307.0745

30. J.D. Barrow, J. Magueijo, Intermediate inflation from rainbow gravity. Phys. Rev. D 88, 103525 (2013). arXiv: 1310.2072

31. Y. Ling, Q. Wu, The big bounce in rainbow universe. Phys. Lett. B 687, 103-109 (2010). arXiv:0811.2615

32. R. Garattini, G. Mandanici, Particle propagation and effective space-time in gravity's rainbow. Phys. Rev. D 85, 023507 (2012). arXiv: 1109.6563
33. R. Garattini, F.S.N. Lobo, Self-sustained wormholes in modified dispersion relations. Phys. Rev. D 85, 024043 (2012). arXiv: 1111.5729

34. A.F. Ali, Black hole remnant from gravitys rainbow. Phys. Rev. D 89, 104040 (2014). arXiv: 1402.5320

35. R. Garattini, Distorting general relativity: gravity's rainbow and $\mathrm{f}(\mathrm{R})$ theories at work. JCAP 1306, 017 (2013). arXiv: 1210.7760

36. R. Garattini, M. Sakellariadou, Does gravitys rainbow induce inflation without an inflaton? Phys. Rev. D 90, 043521 (2014). arXiv: 1212.4987

37. R. Garattini, E.N. Saridakis, Gravitys rainbow: a bridge towards HoavaLifshitz gravity. Eur. Phys. J. C 75, 343 (2015). arXiv: 1411.7257

38. Y. Gim, W. Kim, Thermodynamic phase transition in the rainbow Schwarzschild black hole. JCAP 1410, 003 (2014). arXiv: 1406.6475

39. S.W. Hawking, Particle creation by black holes. Commun. Math. Phys. 43, 199-220 (1975)

40. Y. Ling, X. Li, H.-b. Zhang, Thermodynamics of modified black holes from gravity's rainbow. Mod. Phys. Lett. A 22, 2749-2756 (2007). arXiv:gr-qc/0512084

41. R.C. Tolman, On the weight of heat and thermal equilibrium in general relativity. Phys. Rev. 35, 904-924 (1930)

42. R. Tolman, P. Ehrenfest, Temperature equilibrium in a static gravitational field. Phys. Rev. 36, 1791-1798 (1930)

43. V. P. Frolov, A. Zelnikov, Introduction to Black Hole Physics, Oxford, New York (2011)

44. S.M. Christensen, S.A. Fulling, Trace anomalies and the Hawking effect. Phys. Rev. D 15, 2088-2104 (1977)

45. G. Amelino-Camelia, Quantum-spacetime phenomenology. Living Rev. Rel. 16, 5 (2013). arXiv:0806.0339

46. G. Amelino-Camelia, J.R. Ellis, N.E. Mavromatos, D.V. Nanopoulos, Distance measurement and wave dispersion in a Liouville string approach to quantum gravity. Int. J. Mod. Phys. A 12, 607 624 (1997). arXiv:hep-th/9605211

47. S. Weinberg, Gravitation and Cosmology (Wiley, New York, 1972)

48. Y. Gim, W. Kim, A quantal Tolman temperature. Eur. Phys. J. C 75, 549 (2015). arXiv: 1508.00312

49. H. Boschi-Filho, C.P. Natividade, Anomalies in curved space-time at finite temperature. Phys. Rev. D 46, 5458-5466 (1992)

50. S. Deser, M.J. Duff, C.J. Isham, Nonlocal conformal anomalies. Nucl. Phys. B 111, 45 (1976)

51. W.G. Unruh, Notes on black hole evaporation. Phys. Rev. D 14, 870 (1976)

52. L. Susskind, L. Thorlacius, J. Uglum, The stretched horizon and black hole complementarity. Phys. Rev. D 48, 3743-3761 (1993). arXiv:hep-th/9306069

53. L. Susskind, L. Thorlacius, Gedanken experiments involving black holes. Phys. Rev. D 49, 966-974 (1994). arXiv:hep-th/9308100

54. C.R. Stephens, G’t Hooft, B.F. Whiting, Black hole evaporation without information loss. Class. Quant. Grav 11, 621-648 (1994). arXiv: gr-qc/9310006

55. D. Singleton, S. Wilburn, Hawking radiation, Unruh radiation and the equivalence principle. Phys. Rev. Lett. 107, 081102 (2011). arXiv: 1102.5564 